Instructions for authors, subscriptions and further details:

\title{
http://mcs.hipatiapress.com
}

\section{Using Self-other Differences to Predict College Men's Willingness to Intervene: Assessing the Moderating Effect of Masculine Gender Role Stress}

Josep Maria Canal Barbany ${ }^{1}$

1) Universitat Rovira i Virgili, Spain

Date of publication: October $21^{\text {th }}, 2019$

Edition period: October 2019- February 2020

To cite this article: Canal Barbany, J.M. (2019). Using self-other differences to predict college men's willingness to intervene: Assessing the moderating effect of masculine gender role stress. [Review of the book]. Masculinities and Social Change 8(3), 334-335. doi:

10.17583/MCS.2019.4771

To link this article: http://dx.doi.org/10.4471/MCS.2019.4771

\section{PLEASE SCROLL DOWN FOR ARTICLE}

The terms and conditions of use are related to the Open Journal System and to Creative Commons Attribution License (CC-BY). 


\section{Reviews (II)}

Rice, F. (2017). Using self-other differences to predict college men's willingness to intervene: Assessing the moderating effect of masculine gender role stress. Long Beach, CA: California State University,

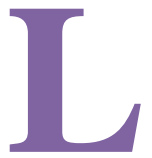

a bystander intervention es una estrategia de prevención de la violencia que ha sido reconocida internacionalmente por su efectividad. En la siguiente tesis se hace especial hincapié en ella pero relacionándola con la predisposición de los hombres jóvenes universitarios a implicarse en la prevención de la violencia. En este sentido, se introducen conceptos interesantes que actúan como variable para entender si afectan en la intervención o no intervención de los hombres. Entre estos conceptos se encuentra el Masculine Gender Role Stress (MGRS) que se entiende como la ansiedad que sufren algunos hombres cuando su masculinidad se está cuestionando.

Es interesante observar como el autor de la tesis pone de manifiesto la investigación existente en el ámbito de la prevención de la violencia en educación superior vinculándola con la construcción de la masculinidad. En este sentido, se evidencia la existencia de resultados que constatan que los hombres son menos propensos a intervenir, siguiendo el patrón de la bystander intervention, cuando existe acoso o violencia hacia las mujeres en los campus universitarios. Las explicaciones que se utilizan para dicha propensión se relacionan con la internalización de las conductas sexistas e hipermasculinas dentro de los círculos de amistad masculina. Desde otra perspectiva teórica a la que se hace referencia en la tesis, la teoría de las normas sociales, se argumenta que la intención de los hombres puede estar afectada por la percepción que tienen alrededor de esta problemática. En otras palabras, el hecho de pensar que los otros hombres no analizan las situaciones de acoso como violencia.

2019 Hipatia Press

ISSN: 2014-3605

DOI: $10.17583 / \mathrm{MCS} .2019 .4771$ 


\section{MCS - Masculinities and Social Change, 8(3) 335}

Con todo este conocimiento, el autor lleva a cabo una investigación con una muestra importante de hombres universitarios. A través de la recogida de datos llega a conclusiones interesantes vinculadas al estado del arte mencionado anteriormente. Sin embargo, introduce una nueva variable que es importante señalar: Self-Other Differences. Esta variable, desde su prisma más positivo, se entiende como aquella creencia en la que las personas suponen que otras personas responden a comportamientos problemáticos más que ellas mismas. De modo que para Rice esta creencia explica en gran parte también el hecho por el cual los hombres presentan un tendencia menor a intervenir ante este tipo de situaciones marcadas por acoso y la violencia. Sin duda, estamos ante una investigación que nos aporta evidencias significativas sobre las barreras existentes para la implementación de la bystander intervention. Partiendo de estos resultados se pueden plantear estrategias para tratar de prevenir dichas dificultades y abordar la socialización de género de las masculinidades en los entornos educativosiquel Missé is a Catalan sociologist and trans activist, reference in Spain and more specifically in Trans activism centered in Barcelona since the 2000's. His contribution to LGBT and Trans studies in Spain has been central to the theorization and defense of Trans Depathologization and the advocacy for trans rights. In addition to his academic work as a researcher at Vic University, he is a trainer and independent public policy consultant for gender and sexual diversity, as well as the promotion of a diverse and critical trans culture.

Josep Maria Canal Barbany josepmaria.canal@estudiants.urv.cat 